\section{THE NORTH AMERICAN POOLED PROJECT (NAPP): POOLED ANALYSES OF CASE-CONTROL STUDIES OF PESTICIDES AND AGRICULTURAL EXPOSURES, LYMPHOHEMATOPOIETIC CANCERS AND SARCOMA}

\begin{abstract}
${ }^{1}$ Manisha Pahwa, ${ }^{2}$ Laura Beane Freeman, ${ }^{3,4}$ John J Spinelli, ${ }^{2}$ Aaron Blair, ${ }^{5,6}$ Punam Pahwa, 5 James A Dosman, ${ }^{7,8}$ John R McLaughlin, ${ }^{1,9}$ Paul A Demers, ${ }^{2}$ Shelia Hoar Zahm, ${ }^{2}$ Kenneth P Cantor, ${ }^{10}$ Dennis D Weisenburger, ${ }^{1}$ Shelley A Harris. ${ }^{1}$ Occupational Cancer Research Centre, Cancer Care Ontario, Toronto, Ontario, Canada; ${ }^{2}$ Division of Cancer Epidemiology and Genetics, US National Cancer Institute, Bethesda, Maryland, USA; ${ }^{3} B C$ Cancer Agency Research Centre, Vancouver, British Columbia, Canada; ${ }^{4}$ School of Population and Public Health, University of British Columbia, Vancouver, British Columbia, Canada; ${ }^{5}$ Canadian Centre for Health and Safety in Agriculture, University of Saskatchewan, Saskatoon, Saskatchewan, Canada; ${ }^{6}$ Community Health and Epidemiology, University of Saskatchewan, Saskatoon, Saskatchewan, Canada; ${ }^{7}$ Public Health Ontario, Toronto, Ontario, Canada; ${ }^{8}$ Samuel Lunenfeld Research Institute, Mount Sinai Hospital, Toronto, Ontario, Canada; ${ }^{9}$ Dalla Lana School of Public Health, University of Toronto, Toronto, Ontario, Canada; ${ }^{10}$ University of Nebraska Medical Center, University of Nebraska, Omaha, Nebraska, USA
\end{abstract}

\subsection{6/oemed-2014-102362.364}

Objectives Previous studies have noted associations between specific pesticides and multiple cancer types. However, assessments for many pesticides have been limited by small numbers of exposed cases. To address this, we established the North American Pooled Project (NAPP), a collaborative effort to evaluate the relationship of pesticide and agricultural exposures to risks of lymphohematopoietic cancers and sarcoma.

Method We harmonised previously collected data from three population-based case-control studies conducted in four American states with a similar Canada-wide study conducted in six provinces. Descriptive analyses of pesticide exposures, personal protective equipment (PPE) use, and demographic data were completed. The prevalence of self-reported pesticide use among cases and controls was determined for specific agents and chemical classes.

Results The NAPP includes 5131 controls and 3274 cases (nonHodgkin lymphoma [NHL] N=1690; Hodgkin lymphoma [HL] $\mathrm{N}=507$; multiple myeloma $[\mathrm{MM}] \mathrm{N}=587$; soft tissue sarcoma $\mathrm{N}=490$ ). Preliminary descriptive analyses indicate that approximately two-thirds of controls and NHL and MM cases ever lived or worked on a farm or ranch. Nearly half of controls and half of NHL, HL, and MM cases reported using any pesticide. Over 120 different insecticides, herbicides, and fungicides were reported. More than $17 \%$ of participants reported using the phenoxy herbicide 2,4-D and over 5\% reported DDT, malathion, atrazine, or glyphosate. Around 6\% of NHL cases and controls reported ever using PPE.

Conclusions The large number of cases and controls and high frequency of pesticide use in the NAPP will allow us to evaluate less commonly used pesticides, cancer sub-types, and smaller relative risks than previously possible.

\section{CUMULATIVE MERCURY EXPOSURE AND PERIPHERAL NERVE FUNCTION IN A SAMPLE OF U.S. DENTAL PROFESSIONALS}

1Julia Anglen, ${ }^{2}$ Leslie Stayner, ${ }^{2}$ Stephen Gruninger. ' University of Illinois of Chicago, Chicago, II, USA; ${ }^{2}$ American Dental Association, Chicago, II, USA

\subsection{6/oemed-2014-102362.365}

Objectives To calculate individual cumulative mercury exposures from a convenience sample of dental professionals and measure the effect on peripheral nerve function.
Method Participants attended the American Dental Association's (ADA) conventions held from 1997-2006. Individual surveys were completed and measurements were taken of the median and ulnar sensory nerve amplitude and latency in the dominant hand. The ADA has measured the average urinary mercury concentration of participants since 1977, allowing a cumulative mercury exposure to be estimated for each individual dentist based on the number of years they practiced dentistry. Both fixed and mixed effects (accounting for repeated measures) linear regression models were used.

Results 3923 observations from 2649 dentists were used to perform linear regression using multiple models. Models included individuals with or without imputed BMI, along with either repeated measures or initial observations only. Adjusted covariates included hand temperature, gender, age and BMI. Individuals with rheumatoid arthritis, diabetes, carpal tunnel syndrome (for median nerve models only), or hand temperatures interfering with the accuracy of the instrument were excluded. The main effect of cumulative exposure was found to be significant ( $\mathrm{p}$-value $<0.05$ ) in median nerve latency and amplitudes but insignificant in ulnar nerve measures. All models but ulnar nerve latency showed a highly significant interaction of cumulative exposure and age ( $\mathrm{p}$-value $<0.01$ ).

Conclusions Using an estimated cumulative mercury exposure as the measure of effect shows a significant positive association with decreased peripheral nerve function. This study is the first of its kind to estimate dentists' cumulative mercury exposure and its effect on peripheral nerve function.

\section{WHEN STANDARD OCCUPATIONAL EXPOSURE ASSESSMENTS ARE UNSUITABLE - EXPOSURE OBSERVATIONS WITH SOUTH AFRICAN HERBICIDE SPRAYERS}

Hanna-Andrea Rother, Federico Andrade Rivas, Michelle DeSouza. University of Cape Town, Cape Town, South Africa

10.1136/oemed-2014-102362.366

Objectives South African herbicide sprayers removing alien vegetation are exposed to a myriad of herbicides resulting in acute and chronic health risks. Workers often are not willing to participate in standard biological monitoring assessments through the provision of blood and urine in order to assess these potential risks. Furthermore, laboratory capacity to analyse herbicides residues are limited. The study aim was to document workers' exposure risks in order to develop health interventions using an observation exposure assessment method.

Method Researchers observed three teams, each comprised of 10 workers and one contractor, from February to June 2012. An observational guide was developed and findings were recorded in a field journal. Observations were supported with video and photographic materials.

Results The on-site observations revealed workers lack of PPE compliance, behaviours that increased their exposure risks, and non-compliance with work standards. Workers' exposure risks were compounded by harsh working conditions, high turnover rates of workers, worker's low risk perceptions, power struggles, and gendered beliefs of masculinity being threatened by PPE use. Conclusions In some circumstances researchers are unable to use biological monitoring methods to establish pesticide exposure risks for workers in developing countries. Observation methods are a viable alternative method, particularly for informing worker risk reduction interventions. 